\title{
Propiedades psicométricas del modelo bifactorial del BDI-II (versión española) en muestras mexicanas de población general y estudiantes universitarios*
}

\author{
Psychometric properties of the BDI-II bifactorial model on \\ mexican general population and university students
}

Recibido: 27 de mayo de 2013 | Revisado: 29 de mayo de 2014 | Aceptado: 17 de octubre de 2014

\author{
Benito Daniel Estrada Aranda ** \\ Universidad Autónoma de San Luis Potosí, México \\ Carmen Delgado Álvarez *** \\ Universidad Pontificia de Salamanca, España \\ RENÉ LANDERO HERNÁNDEZ ***** \\ MÓnICA Teresa GONZÁlez RAMÍREZ***** \\ Universidad Autónoma de Nuevo León, México
}

doi: 10.11144/Javeriana.upsy14-1.ppmb

Para citar este artículo: Estrada, B. D., Delgado, C., Landero, R., \& González, M. T. (2015). Propiedades psicométricas del modelo bifactorial del BDI-II (versión española) en muestras mexicanas de población general y estudiantes universitarios. Universitas Psychologica, 14(1), xx-xx. http://dx.doi.org/10.11144/ Javeriana.upsy14-1.ppmb

Artículo original resultado de investigación

** Profesor-Investigador. Departamento: Posgrado de la Facultad de Psicología. Contacto principal para correspondencia. Correo electrónico: benito. estrada.aranda@gmail.com

*** $\quad$ Profesora titular Facultad de Psicología. Correo electrónico: cdelgado@usal.es

***** Profesor-Investigador. Facultad de Psicología. Correo electrónico: rlandero1_mx@yahoo.com. $\mathrm{mx}$

******* Profesora-Investigadora. Facultad de Psicología. Correo electrónico: monygzz77@yahoo.com

\section{RE S U MEN}

En este trabajo, se analizan las propiedades psicométricas de la versión española (Sanz, Navarro, \& Vázquez, 2003) del Inventario para la Depresión de Beck ([BDI-II]; Beck, Steer, \& Brown, 1996) en muestras mexicanas no clínicas: 474 estudiantes universitarios y 478 personas de población general. La consistencia interna resultó satisfactoria en ambas muestras siendo alfa de Cronbach 0.901 para la muestra total. Los resultados coinciden con los reportados en estudios previos españoles (Sanz, Navarro \& Vázquez, 2003; Sanz, Perdigón \& Vazquez, 2003) acerca de las propiedades psicométricas del instrumento, así como otros estudios internacionales revisados. El análisis factorial confirmatorio muestra buenos índices de ajuste al modelo bifactorial de la escala. Se encontraron diferencias significativas en las puntuaciones en función del sexo, pero no se observan efectos significativos de la edad. Se concluye que la versión española del BDI-II puede ser un instrumento válido y fiable para evaluar la sintomatología depresiva en la población general adulta mexicana y en estudiantes universitarios.

Palabras clave

BDI-II; propiedades psicométricas en México; depresión; población general; estudiantes universitarios

\section{A B S T R A C T}

The purpose of this study was to test the Spanish version (Sanz, Navarro, \& Vazquez, 2003) of the Beck Depression Inventory-II ([BDI-II]; Beck, Steer, $\&$ Brown, 1996) in the Mexican general population and undergraduate university students on non clinical samples. The reliability and construct validity obtained in a sample of 474 university students and 478 people from the general population, confirmed good psychometric properties: Cronbach alpha of 0.901 and Confirmatory factor analysis, showed good adjustment indices to two-factor model scale. These results agree with those reported in previous studies both international and Spanish studies on the psychometric properties of the instrument (Sanz, Navarro, \& Vázquez, 2003; Sanz, Perdigón \& Vázquez, 2003). Confirmatory factor analysis showed a good fit of the Mexican population data, the two-factor model reported in the Spanish population. Finally, the distributions of scores were similar to those found in 
Spanish studies. There were significant differences in scores by gender, but there are no significant effects of age. It is concluded that the Spanish version of the BDI-II may be a valid and reliable instrument to assess depressive symptoms in college students and the general population of Mexican adults. Keywords

BDI-II; psychometric properties; depression; Mexican general population; university students

\section{Introducción}

El Inventario de Depresión de Beck ([BDI]; Beck, Steer, \& Brown, 1996) ha sido ampliamente utilizado, tanto en investigación psicológica como en evaluación clínica de la intensidad de los síntomas depresivos, siendo replicado y adaptado a numerosas lenguas y culturas (Beck, Steer, \& Brown, 1996; Byrne, Stewart, \& Lee, 2004; Canel-Çinarbas, Cui, \& Lauridsen, 2011; Campos \& Gonçalves, 2012; Dozois, Dobson, \& Ahnberg, 1998; Jakšić, Ivezić, Jokić-Begić, Surányi, \& Stojanović-Špehar, 2013; Kojima et al., 2002; Song, Lee, Kim, \& Lee, 2012; Steer \& Clark, 1997; Whisman, Perez, \& Ramel, 2000).

A diferencia de otros instrumentos, el BDI ha sido revisado en tres ocasiones para adaptarlo a los sucesivos cambios introducidos en el diagnóstico del trastorno depresivo mayor. Las revisiones del DSM-III y del DSM-IV (American Psychiatric Association [APA], 1987, 1994) llevaron a Beck y sus colegas a realizar cambios en los contenidos de la primera versión (BDI-I) para adaptarlo a los nuevos criterios diagnósticos. La primera revisión dio lugar al BDI-IA (Beck, Rush, Shaw, \& Emery, 1979; Beck \& Steer, 1993; Beck, Steer, Ball, \& Ranieri, 1996). Esta segunda versión revisada en 1978 (BDI-IA) fue una de las más utilizadas, tanto en pacientes psiquiátricos como en población general (Archer, Maruish, Imhof, \& Piotrowski, 1991; Piotrowski \& Keller, 1992; Piotrowski, Sherry, \& Keller, 1985).

El BDI-IA, actualmente en desuso, fue adaptado en su día a población mexicana, con resultados satisfactorios: el estudio de Torres-Castillo, Hernández-Malpica y Ortega-Soto (1991) con pacientes con enfermedades cardiovasculares, el de SuárezMendoza, Cardiel, Caballero-Uribe, Ortega-Soto, \& Marquez-Marin, M. (1997) con pacientes con artritis reumatoide y el de Jurado et al. (1998) con muestras de población general y estudiantes universitarios. No obstante, todos ellos presentaban el inconveniente de utilizar versiones en español provenientes de traducciones realizadas en los Estados Unidos. Años más tarde, Beck, Steer y Brown (1996) publicaron una nueva versión del Inventario para la Depresión de Beck-II (BDI-II), versión actualmente vigente, que aporta algunas modificaciones importantes en relación con sus versiones anteriores.

En España, se realizaron varias adaptaciones del BDI en sus diferentes versiones (Conde, Esteban, \& Useros, 1976; Montorio, 1990; Sanz \& Vázquez, 1998; Vázquez \& Sanz, 1991, 1997, 1999). La última versión del BDI-II fue adaptada a la población española tanto con estudiantes universitarios, siguiendo un cuidadoso proceso de traducción que implicó varias etapas y varios traductores bajo la metodología back translation y un estudio piloto de su aplicabilidad y de la comprensibilidad de sus instrucciones e ítems y análisis de sus propiedades psicométricas (Sanz, Navarro, \& Vázquez, 2003), así como con una muestra de la población general (Sanz, Perdigón, \& Vázquez, 2003) y muestras clínicas (Sanz, GarcíaVera, Espinosa, Fortín, \& Vázquez 2005).

Estudios más recientes confirman la adecuación psicométrica de la versión española del BDI-II en muestras de distintas características: población general, estudiantes universitarios, estudiantes de secundaria y muestras psicopatológicas (Sanz \& García-Vera, 2009). Sin embargo, la validación en muestras con ciertas patologías médicas, como el estudio realizado por Del Pino et al. (2012) con pacientes coronarios, ofrece datos contradictorios en su estructura factorial, al reportar una estructura de un solo factor.

Los intentos de adaptación del BDI-II a población mexicana (Novy, Stanley, Averill, \& Danza, 2001; Penley, Wiebe, \& Nwosu, 2003) fueron realizados con una versión en español generada por la editorial The Psychological Corporation, de la que no se ofrecen datos de fiabilidad y validez ni datos normativos para población mexicana (Sierra, 2004). Por otro lado, las muestras de estos estudios presentan características que las hacen inadecuadas para generalizar los resultados a población mexicana. La muestra utilizada en el estudio de Novy 
et al. incluía personas adultas nacidas en Estados Unidos, América del Sur, Centroamérica y México y diagnosticadas con trastorno de ansiedad. Sus resultados mostraron buena consistencia interna $(\alpha$ $=0.94)$ y correlación alta con la versión original en inglés del BDI-II $(r=0.94)$ para esta muestra heterogénea. La utilizada por Penley et al. incluía 122 pacientes mexicano-estadounidenses con problemas renales que recibían un tratamiento de hemodiálisis, obteniendo un alfa de Cronbach de 0.92 también satisfactorio para este tipo de pacientes. Solo el estudio posterior de Wiebe y Penley (2005) utilizó una muestra de estudiantes universitarios, obteniendo datos de consistencia interna satisfactorios, tanto de la versión original en inglés $(\alpha=$ 0.89) como de su versión en español $(\alpha=0.91)$, y una equivalencia en la estructura factorial de ambas versiones; pero las características de la muestra (mexicanos residentes en EE. UU.) también limita la generalización a población mexicana.

Otro intento de adaptación, fue el realizado por Sierra (2004) quien aplicó la versión española del BDI-II (Sanz, Perdigón et al., 2003) a una muestra de 202 estudiantes mexicanos, consiguiendo buena consistencia interna $(\alpha=0.89)$. La obtención de un factor general en la matriz sin rotar, mediante análisis factorial exploratorio, presenta dos inconvenientes para comparar sus resultados con los originales de la versión española. Por un lado, la unidimensionalidad reportada en el análisis factorial exploratorio es la solución esperada cuando no se rota la matriz. Por otro lado, este análisis exploratorio no permite contrastar estructuras factoriales, por lo que no aporta datos sobre el ajuste de la población mexicana a la estructura bifactorial. Wilson y Blumentritt (2007) realizaron otro estudio con muestras clínicas de adolescentes méxico-americanos, confirmando buena consistencia interna $(\alpha=0.9)$ y evidencias satisfactorias de validez convergente y divergente, a partir de las correlaciones obtenidas con otras escalas clínicas. La estructura factorial reportada confirmó dos factores en la escala, uno cognitivo-somático y otro afectivo, en muestras con las características señaladas; pero no se dispone de estudios con muestras de población general. La revisión de estudios de adaptación del BDI-II a población mexicana muestra, por tanto, que no se dispone hasta el momento de estudios publicados que confirmen las propiedades psicométricas de la versión española del BDI-II (Vázquez, Sanz, \& Perdigón, 2003), en población no clínica.

El interés de adaptar la versión española del BDIII a otros países hizo que fuera aplicada a muestras de adolescentes femeninas chilenas (Cova, Rincón, \& Melipillán, 2009) reportando índices de consistencia interna alfa de Cronbach de 0.93. Como resultado, la adecuación reportada fue utilizada (Cova, Rincón, Inostroza, \& Melipillán, 2012) para analizar prospectivamente la relación entre soporte familiar, estilo inferencial depresogénico y rumiación negativa con sintomatología depresiva en adolescentes chilenas.

La relevancia del BDI-II y la generalización de su uso, tanto en contextos de investigación como en la práctica de evaluación clínica, ha sido puesta de relieve en la reciente revisión realizada por $\operatorname{Sanz}$ (2011), siendo la escala más utilizada en las publicaciones científicas sobre depresión y superando en más del doble-frente a la escala situada en segundo lugar-el número de artículos que la utilizan para evaluar este trastorno. El BDI-II se ha convertido, por tanto, en el instrumento de autoinforme más utilizado y más investigado para evaluar la depresión, como refiere el estudio citado. Por otro lado, su relevancia no se circunscribe al ámbito de la depresión, ya que en la práctica profesional general, ocupa el tercer lugar entre los tests más utilizados en la práctica clínica española (Muñiz \& Fernández-Hermida, 2010). Su reciente adaptación a la lengua de signos española (Estrada, 2008; Estrada, Delgado, \& Beyebach, 2010) y a la lengua de señas mexicana (Estrada $\&$ Ruíz, 2012) ha hecho posible también su utilización en la evaluación clínica de personas sordas, convirtiéndose en uno de los instrumentos de autoinforme más ampliamente utilizado.

Por todo lo anteriormente expuesto, se plantea la necesidad de analizar la adecuación psicométrica de la versión española del Inventario de Depresión de Beck (BDI-II) para población mexicana, así como analizar si se confirman las diferencias entre mujeres y hombres, reportadas en otros países. Sobre este aspecto, los estudios previos en población 
mexicana, a los que se ha aludido, ofrecen resultados contradictorios. Sierra (2004) no obtiene diferencias significativas, que sí se obtienen con la versión original estadounidense (Beck, Steer, \& Brown, 1996; Steer, Ball, Ranieri, \& Beck, 1999) y con la versión española (Sanz, Perdigón et al., 2003), siendo las mujeres quienes dan puntuaciones superiores.

Los objetivos de este estudio responden pues a un doble interés. Por un lado, siguiendo la reciente recomendación de la Association for Psychological Science (2013), el interés teórico de replicar estudios previos que permitan confirmar en otras muestras investigaciones ya publicadas. Por otro lado, el interés aplicado de disponer de datos de población no clínica, no reportados hasta el momento, que avalen la utilización de la versión española del BDI-II en población mexicana. Este objetivo se concreta en la evaluación del ajuste del BDI-II al modelo bifactorial en población mexicana, la estimación de la consistencia interna de las subescalas y escala total (confiabilidad) y analizar diferencias en las puntuaciones por género.

\section{Método}

\section{Participantes y procedimiento}

Participaron en el estudio 975 sujetos de la ciudad de Monterrey en Nuevo León (México), que accedieron a participar voluntariamente. El método de muestreo fue no probabilístico incidental, como es usual en este tipo de estudios, quedando integrada la muestra final por 952 sujetos tras eliminar 23 casos atípicos, detectados por el método de Mahalanobis al cuadrado (impacto sobre la variable entre 0.075 y 0.114). La distribución por submuestras fue 474 estudiantes universitarios y 478 participantes de la población general. La muestra de estudiantes universitarios cursaba estudios de áreas de conocimiento diversas: Artes Visuales $(\mathrm{N}=44)$, Comunicación $(N=46)$, Contaduría Pública y Administración $(N=39)$, Filosofía y Letras $(N=$ $45)$, Medicina $(N=48)$, Odontología $(N=50)$, Derecho y Criminología $(N=44)$, Salud Pública y Nutrición $(N=44)$, Psicología $(N=83)$. Los participantes aceptaron su participación mediante una carta de consentimiento informado, antes de responder los cuestionarios. En la Tabla 1 se muestran los índices descriptivos por edad y sexo.

\section{Instrumento}

La escala BDI-II elaborada por Beck, Steer y Brown (1996) adaptada a población española por Sanz, Navarro et al. (2003) es un instrumento de autoinforme para evaluar la intensidad de la sintomatología depresiva, mediante 21 ítems politómicos con cuatro alternativas de respuesta ordenadas de menor a mayor gravedad de los síntomas. En esta aplicación en muestras de la población mexicana, el BDI-II (Sanz, Navarro et al., 2003) no requirió de ninguna adaptación lingüística cultural del español de España al hablado en México, ya que no presentaba diferencias culturales en un sentido semántico. La valoración de los ítems se realiza a partir del estado de ánimo de los últimos 15 días, incluyendo el día en que se responde. El rango de edad de su aplicación es de 13 a 65 años. La puntuación total en la escala oscila entre 0 (puntuación mínima) y 63 (puntuación máxima). La escala evalúa una dimensión general integrada por todos los ítems y contiene dos dimensiones altamente correlacionadas: (a) dimensión cognitivo-afectiva de 12 ítems $(1,2,3$, $5,6,7,8,9,10,11,13$ y 17) y (b) dimensión somático-

Tabla 1

Descriptivos de edad y sexo de la muestra total y submuestras mexicanas

\begin{tabular}{lccccccccc}
\hline \multirow{2}{*}{ Muestra } & \multirow{2}{*}{$\mathrm{N}$} & \multicolumn{9}{c}{ Edad } & \multicolumn{2}{c}{ Sexo } \\
\cline { 3 - 10 } & & Mín. & Máx. & Media & Sx & As & K & Mujeres (\%) & Hombres (\%) \\
\hline Estudiantes & 474 & 17 & 28 & 20.45 & 2.17 & +0.68 & +0.53 & 67.3 & 32.7 \\
General & 478 & 13 & 65 & 27.33 & 14.06 & +0.64 & -1.08 & 65.7 & 34.3 \\
\hline Total & 952 & 13 & 65 & 23.91 & 10.64 & +1.57 & +1.46 & 66.5 & 33.5 \\
\hline
\end{tabular}

Fuente: elaboración propia 
motivacional de 7 ítems $(4,12,15,16,18,20$ y 21$)$. Se tomaron los mismos puntos de corte que Beck, Steer y Brown (1996) plantearon, y que también fueron usados en los tres estudios españoles de adaptación del BDI-II, ya mencionados.

\section{Resultados}

Distribución de puntuaciones de sintomatología depresiva

La distribución de puntuaciones de sintomatología depresiva resultó similar a la reportada en población española, como se muestra en la Tabla 2.

Los valores de la puntuación total en la escala (factor general) oscilaron en la población mexicana entre 0 y 52 puntos, con media igual a 9.18 (9.3 para estudiantes y 9.1 para población general) y desviación típica igual a 8.29 (7.8 para estudiantes y 8.7 para población general). Conforme a lo esperado en muestras no clínicas, la distribución resultó leptocúrtica $(\mathrm{K}=$ +4.08 ) indicando mayor concentración de puntuaciones en un rango de valores más restringido y asimétrica positiva (As $=+1.739$ ), indicando frecuencias altas en los valores bajos del rango de respuesta.

\section{Descriptivos y homogeneidad de los ítems}

Siguiendo las recomendaciones sobre el uso del BDI-II en investigación (Sanz, 2011), se ofrecen datos sobre las propiedades de los ítems. Las puntuaciones medias oscilaron entre 0.17 (ítem 9: pensamientos de suicidio) y 0.85 (ítem 16 : cambios en el sueño). El carácter no clínico de las muestras afecta, como era de esperar, a la simetría y curtosis de la práctica totalidad de los ítems. Se obtiene asimetría positiva y leptocurtosis para la mayoría de los ítems, ya que la sintomatología depresiva se concentra en valores mínimos o leves (asimetría positiva) con poca variabilidad y apuntamiento de las distribuciones (leptocurtosis), siendo los resultados muy similares a los obtenidos en muestras españolas (Sanz, Navarro et al., 2003; Sanz, Perdigón et al., 2003). No se realizaron transformaciones en los datos, asumiendo la robustez a la violación del supuesto de normalidad por el alto tamaño muestral (Hair, Anderson, Tatham, \& Black, 2001). Todos los ítems mostraron buenos índices de homogeneidad corregida, por lo que su discriminación puede considerarse satisfactoria (Raykov \& Marcoulides, 2011).

\section{Consistencia interna}

La consistencia interna en la muestra total mexicana resultó satisfactoria, tanto para el factor general $(\propto=0.9)$, como para cada uno de los factores: 0.85 en la dimensión cognitivo-afectiva y 0.78 en la dimensión somático-motivacional. Los índices obtenidos, diferenciando población de estudiantes y población general, resultaron igualmente satisfactorios y similares a los referidos en población española como se muestra en la Tabla 4.

\section{Estructura factorial}

El ajuste a la estructura bifactorial de la versión española del BDI-II (Sanz, Perdigón et al., 2003; Sanz, Navarro et al., 2003) de los datos de la pobla-

TABLA 2

Distribución de sintomatología depresiva

\begin{tabular}{lcccccccc}
\hline & \multicolumn{3}{c}{ Estudiantes } & \multicolumn{4}{c}{ Población General } \\
\hline \multirow{2}{*}{$\begin{array}{l}\text { Niveles de Depresión } \\
\text { (Beck et al., 1996) }\end{array}$} & $\begin{array}{c}\text { Población española } \\
\text { N }=590\end{array}$ & \multicolumn{2}{c}{\begin{tabular}{c} 
Población mexicana \\
\cline { 2 - 8 }
\end{tabular}} & \multicolumn{2}{c}{$\mathrm{N}=474$} & \multicolumn{2}{c}{$\begin{array}{c}\text { Población española } \\
\mathrm{N}=470\end{array}$} & \multicolumn{2}{c}{$\begin{array}{c}\text { Población mexicana } \\
\mathrm{N}=478\end{array}$} \\
\hline Minima (0-13) & $\mathrm{f}$ & $\%$ & $\mathrm{f}$ & $\%$ & $\mathrm{f}$ & $\%$ & $\mathrm{f}$ \\
Leve (14-19) & & & 77.4 & 373 & 75.3 & 354 & 76.4 & 359 \\
Moderada (20-28) & & & 14.5 & 70 & 15.7 & 74 & 11.7 & 55 \\
Grave (29-63) & & 5.2 & 25 & 5.7 & 27 & 7.7 & 36 \\
\hline
\end{tabular}

Fuente: elaboración propia 
Benito Daniel Estrada Aranda, Carmen Delgado Álvarez,

René Landero Hernández, Mónica Teresa González Ramírez

Tabla 3

Descriptivos e índices de homogeneidad de los items en población mexicana

\begin{tabular}{lccccc}
\hline & Media & Desviación estándar & Asimetría & Curtosis & Homogen. corregida \\
\hline 1. Triste & 0.38 & 0.723 & 1.762 & 2.036 & 0.592 \\
2. Pesimista & 0.29 & 0.63 & 2.514 & 6.522 & 0.506 \\
3. Fracaso & 0.24 & 0.528 & 2.326 & 5.567 & 0.545 \\
4. Pérdida de placer & 0.42 & 0.639 & 1.393 & 1.37 & 0.553 \\
5. Culpa & 0.44 & 0.592 & 1.33 & 2.552 & 0.539 \\
6. Castigo & 0.36 & 0.723 & 2.387 & 5.563 & 0.448 \\
7. Insatisfecho & 0.37 & 0.712 & 2.016 & 3.538 & 0.574 \\
8. Autocritica & 0.66 & 0.784 & 1.067 & 0.627 & 0.555 \\
9. Pensamientos de suicidio & 0.17 & 0.433 & 2.881 & 9.944 & 0.485 \\
10. Llanto & 0.49 & 0.879 & 1.788 & 2.156 & 0.549 \\
11. Agitación & 0.46 & 0.737 & 1.828 & 3.281 & 0.473 \\
12. Pérdida de Interés & 0.4 & 0.634 & 1.74 & 3.388 & 0.564 \\
13. Indecisión & 0.39 & 0.714 & 2.088 & 4.245 & 0.517 \\
14. Inutilidad & 0.26 & 0.584 & 2.367 & 5.182 & 0.54 \\
15. Débil, flojo & 0.55 & 0.642 & 0.906 & 0.4 & 0.561 \\
16 Cambios en el sueño & 0.85 & 0.763 & 0.793 & 0.57 & 0.433 \\
17. Irritabilidad & 0.37 & 0.629 & 1.921 & 4.215 & 0.569 \\
18. Cambios en apetito & 0.65 & 0.781 & 1.257 & 1.411 & 0.501 \\
19. Dificultad concentración & 0.64 & 0.748 & 0.949 & 0.23 & 0.569 \\
20. Cansancio o fatiga & 0.54 & 0.691 & 1.218 & 1.365 & 0.578 \\
21. Pérdida interés sexo & 0.25 & 0.6 & 2.653 & 6.953 & 0.393 \\
\hline
\end{tabular}

Fuente: elaboración propia

Tabla 4

Consistencia interna en población española y mexicana

\begin{tabular}{lcccc}
\hline & \multicolumn{2}{c}{ Estudiantes } & \multicolumn{2}{c}{ Población General } \\
\hline $\begin{array}{l}\text { Consistencia interna } \\
\alpha \text { de Cronbach }\end{array}$ & $\begin{array}{c}\text { Población española } \\
\mathrm{N}=590\end{array}$ & $\begin{array}{c}\text { Población mexicana } \\
\mathrm{N}=474\end{array}$ & $\begin{array}{c}\text { Población española } \\
\mathrm{N}=470\end{array}$ & $\begin{array}{c}\text { Población mexicana } \\
\mathrm{N}=478\end{array}$ \\
\cline { 2 - 5 } BDI-II versión española & $\alpha$ & $\alpha$ & $\alpha$ & $\alpha$ \\
\hline Factor general & 0.89 & 0.88 & 0.87 & 0.91 \\
Dimensión cognitivo-afectiva & (n. d.) & 0.85 & (n. d.) & 0.86 \\
Dimensión somático-motivacional & (n. d.) & 0.74 & (n. d.) & 0.81 \\
\hline
\end{tabular}

Fuente: elaboración propia

ción mexicana se realizó mediante análisis factorial confirmatorio (Jöreskog \& Sörbom, 1993) para cada una de las muestras mexicanas (estudiantes y población general) y para la muestra total. Se utilizó el programa estadístico Amos en su versión 6.0 (Arbuckle, 2005), obteniéndose los parámetros a través del método de Maximum Likelihood, utilizando la matriz de covarianzas entre los ítems como input para el análisis, y la técnica bootstrapping para con- trolar la no normalidad de los datos. Para establecer la bondad de ajuste, se tomaron los índices usuales en este tipo de estudios (Gallardo \& Moyano-Díaz, 2012), tal como se muestran en la tabla siguiente y siguiendo las recomendaciones de diferentes autores (Bentler, 1990; Bollen \& Long, 1993; McDonald \& Marsh, 1990; Mulaik et al., 1989).

Los índices FIT obtenidos revelan, por tanto, un buen ajuste de los datos mexicanos al modelo 
TABLA 5

Índices de ajuste al modelo bifactorial de la versión española del BDI-II

\begin{tabular}{lcccc}
\hline \multicolumn{1}{c}{ Índices FIT } & Valores de ajuste & Estudiantes & Población General & Total \\
\hline CMIN/df & $<5$ & $2.8^{*}$ & $2.728^{*}$ & $3.884^{*}$ \\
GFI & $>0.9$ & $0.91^{*}$ & $0.913^{*}$ & $0.936^{*}$ \\
RMSEA & $<0.07$ & $0.062^{*}$ & $0.06^{*}$ & $0.055^{*}$ \\
RMSR & $<0.07$ & $0.024^{*}$ & $0.022^{*}$ & $0.019^{*}$ \\
CFI & $\geq 0.85$ & $0.886^{*}$ & $0.911^{*}$ & $0.915^{*}$ \\
IFI & $\geq 0.85$ & $0.887^{*}$ & $0.911^{*}$ & $0.915^{*}$ \\
\hline
\end{tabular}

* Buen ajuste.

Fuente: elaboración propia

bifactorial de la versión española del BDI-II, tanto para cada una de las submuestras (estudiantes y población general) como para la muestra total. La correlación entre ambos factores fue de 0.898 (0.872 en estudiantes universitarios y 0.919 en población general).

\section{Efecto de variables sociodemográficas}

En cuanto al efecto de la edad y el sexo, el análisis de regresión simple mostró que la edad no tiene efectos significativos $(p=0.244)$, pero sí el sexo cuyos efectos sobre la puntuación general, se analizaron mediante ANOVA $(p=0)$, obteniendo puntuaciones significativamente mayores en las mujeres, en contra de lo reportado por Sierra (2004) y confirmando los resultados obtenidos por Sanz, Perdigón et al. (2003) en población española.

El efecto de interacción observado entre sexo y tipo de población mexicana $(p=0.01)$ muestra que las diferencias entre mujeres y hombres son mayores en la muestra de población general que en la muestra de estudiantes, como se presenta en el gráfico siguiente:

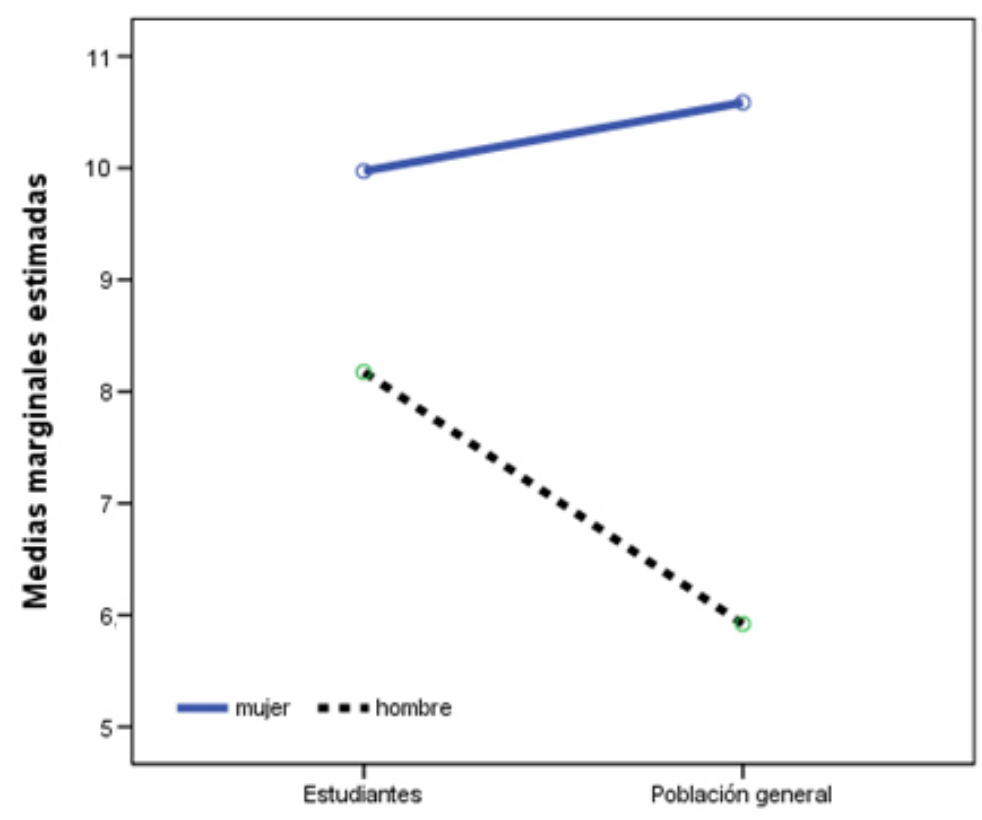

Figura 1. Diferencias por sexo y tipo de población.

Fuente: elaboración propia 


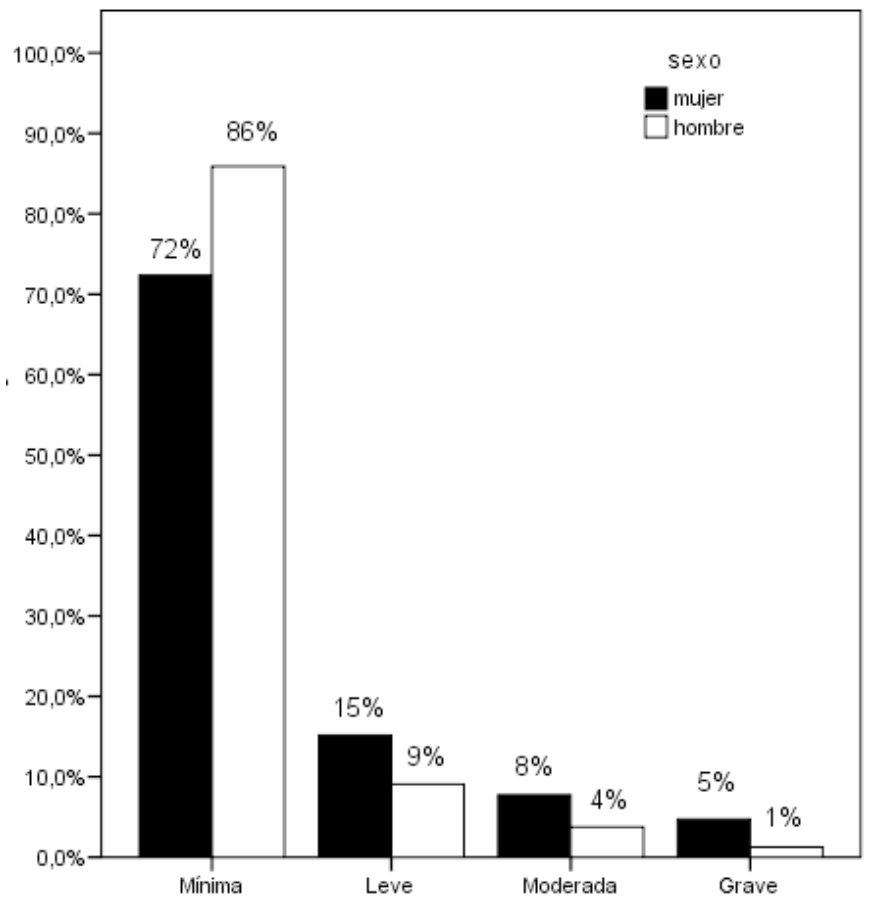

Figura 2. Sintomatología depresiva por sexo.

Fuente: elaboración propia

La sintomatología depresiva en población mexicana es significativamente mayor en mujeres que en hombres, pero estas diferencias por sexo son menores en la muestra de estudiantes universitarios que en la población general. El gráfico siguiente muestra los porcentajes de sintomatología depresiva por sexo:

Los descriptivos por sexo y tipo de población (general vs. estudiantes universitarios) se pueden observar en la tabla siguiente.

\section{Discusión}

El análisis de las propiedades psicométricas del modelo bifactorial de la versión española del BDIII en población mexicana confirma, ante todo, una distribución de puntuaciones de sintomatología depresiva en población no clínica, similar a la encontrada en otros países. Las propiedades psicométricas de la versión española del BDI-II, analizadas en este estudio, permiten concluir so-

TABLA 6

Descriptivos del BDI-II en población mexicana por sexo y muestra

\begin{tabular}{llccc}
\hline \multicolumn{1}{c}{ Sexo } & \multicolumn{1}{c}{ Muestra } & M & DT & N \\
\hline \multirow{2}{*}{ Mujer } & Estudiantes & 9.9718 & 7.82 & 319 \\
& Población General & 10.5860 & 9.49 & 314 \\
& Total & 10.2765 & 8.68 & 633 \\
\hline \multirow{2}{*}{ Hombre } & Estudiantes & 8.1742 & 7.87 & 155 \\
& Población General & 5.9207 & 5.73 & 164 \\
& Total & 7.0157 & 6.93 & 319 \\
\hline \multirow{2}{*}{ Total } & Estudiantes & 9.3840 & 7.87 & 474 \\
& Población General & 8.9854 & 8.67 & 478 \\
& Total & 9.1838 & 8.28 & 952 \\
\hline
\end{tabular}

Fuente: elaboración propia 
bre su adecuación para ser utilizada en población no clínica mexicana: estudiantes universitarios y población general. Esta confirmación aporta evidencia empírica que justifica la utilización de este instrumento en población mexicana, dando soporte psicométrico a los estudios que utilizan el instrumento y que hasta el momento no disponían de evidencias que justificaran la adecuación de la escala para soportar las inferencias realizadas, a partir de las puntuaciones obtenidas en esta población.

Respecto a la fiabilidad, la versión española del BDI-II obtuvo una buena consistencia interna en población mexicana (0.9) similar a la obtenida en estudios que han usado versiones del BDI-II en español (Sanz, Navarro et al., 2003; Sanz, Perdigón et al., 2003; Sanz et al., 2005; Novy et al., 2001; Penley et al., 2003). Estos resultados son congruentes con los obtenidos en sus respectivas lenguas de signos, derivadas de versiones del BDI-II en español: $\alpha$ $=0.88$ en lengua de signos española (Estrada et al., 2010) y $\alpha=0.9$ en lengua de señas mexicana (Estrada \& Ruíz, 2012). Estos resultados son igualmente congruentes con los obtenidos en población méxico-americana en Estados Unidos (Novy et al., 2001; Penley et al., 2003) y en población chilena (Cova et al., 2009).

En cuanto a la estructura de dos factores correlacionados propuesta para el BDI-II (Beck et al., 1996), se obtiene un buen ajuste a la estructura de la versión española, tanto en la muestra de estudiantes mexicanos como en la de población general, pudiendo considerar por tanto que el modelo bifactorial de la versión española del BDI-II es adecuado para la población mexicana. Se confirman dos factores identificables, interrelacionados entre sí: un factor cognitivo-afectivo de 13 ítems y un factor somático-motivacional de 8 ítems. Estos resultados confirman en población mexicana lo obtenido en muestras de otros países tanto con la versión original en inglés (Beck et al., 1996; Canel-Çinarbas et al., 2011; Dozois et al., 1998; Kojima et al., 2002; Novy et al., 2001; Penley et al., 2003; Steer \& Clark, 1997; Whisman et al., 2000) como con la versión española (Del Pino et al., 2012; Estrada et al., 2010; Estrada \& Ruíz,
2012; Sanz, Navarro et al., 2003; Sanz, Perdigón et al., 2003; Sanz et al., 2005). Los resultados de este estudio, avalan por un lado el carácter transcultural del constructo depresión operacionalizado en el BDI-II, cuya estructura interna incluye dos dimensiones diferenciadas, altamente correlacionadas entre sí. Por otro lado, además de su carácter transcultural, los resultados aportan nueva evidencia empírica sobre la adecuación de la definición bifactorial del constructo para población no clínica.

Por último, al encontrarse que la sintomatología depresiva es significativamente mayor en las mujeres de la población general en México, se confirma lo que se ha reportado en España en población general (Sanz, Perdigón et al., 2003) y en estudiantes universitarios españoles (Sanz, Navarro et al., 2003), así como en la literatura previa antes señalada.

Existen algunas limitaciones en este estudio que deben ser tenidas en cuenta, pudiendo señalar en primer lugar el carácter no aleatorio de las muestras, puesto que fueron incluidas en el estudio por su accesibilidad, como es usual en este tipo de investigaciones. Por otro lado, aunque no hay elementos que hagan pensar que la población de Monterrey tenga características diferentes al resto del país, sería deseable replicar el estudio con participantes de otras regiones, con el fin de garantizar la generalización de los datos a la población mexicana, de un modo más riguroso. Se concluye, por tanto, que la versión española del BDI-II replica la consistencia interna y la estructura bifactorial (cognitivo-afectivo y somático motivacional), obtenidas en población española, justificando su uso en población mexicana no clínica.

\section{Referencias}

American Psychiatric Association (1980). Diagnostic and statistical manual of mental disorders (3.a ed.). Washington, DC: Autor.

American Psychiatric Association (1987). Diagnostic and statistical manual of mental disorders (3.a ed., rev.). Washington, DC: Autor. 
American Psychiatric Association. (1994). Diagnostic and statistical manual of mental disorders (4.a ed.). Washington, DC: Autor.

Arbuckle, J. L. (2005). Amos 6.0 User's Guide. Chicago, IL: SPSS Inc.

Archer, R. P., Maruish, M., Imhof, E. A., \& Piotrowski, C. (1991). Psychological test usage with adolescent clients: 1990 survey findings. Professional Psychology: Research and Practice, 22(3), 247-252.

Association for Psychological Science. (2013). Registered Replication Report. http://www.psychologicalscience.org/index.php/replication

Beck, A. T., Rush, A. J., Shaw, B. F., \& Emery, G. (1979). Cognitive therapy of depression (Desclée de Brower, Trad.). New York: Guilford Press.

Beck, A. T. \& Steer, R. A. (1993). Beck Depression Inventory. Manual. San Antonio, TX: The Psychological Corporation.

Beck, A. T., Steer, R. A., Ball, R., \& Ranieri, F. (1996). Comparison of Beck Depression Inventories -IA and -II in psychiatric outpatients. Journal of Personality Assessment, 67(3), 588-597.

Beck, A. T., Steer, R. A., \& Brown, G. K. (1996). Manual for the Beck Depression Inventory-II. San Antonio, TX: Psychological Corporation.

Bentler, P. M. (1990). Comparative fit indexes in structural models. Psychological Bulletin, 107(2), 238-246.

Bollen, D. A., \& Long, J. S. (1993). Testing structural equation models. Newbury Park, CA: Sage.

Byrne, M. B., Stewart, M. S., \& Lee, W. P. (2004). Validating the Beck Depression Inventory-II for Hong Kong community adolescents. International Journal of Testing, 4(3), 199-216. doi:10.1207/ s15327574ijt0403_1

Campos, C. R., \& Gonçalves, B. (2012). The Portuguese version of the Beck Depression Inventory-II (BDIII): Preliminary psychometric data with two nonclinical samples. European Journal of Psychological Assessment, 27(4), 258-264. doi:10.1027/1015-5759/ a000072

Canel-Çinarbas, D., Cui, Y., \& Lauridsen, E. (2011). Cross-cultural validation of the Beck Depression Inventory-II across U.S. and Turkish Samples. Measurement and Evaluation in Counseling and Development, 44(2), 77-91 doi: 10.1177/0748175611400289
Conde, V., Esteban, T., \& Useros, E. (1976). Revisión crítica de la adaptación castellana del Cuestionario de Beck. Revista de Psicología General y Aplicada, 31, 469-497.

Cova, S. F., Rincón, G. P., \& Melipillán, A. R. (2009). Reflexión, rumiación negativa y desarrollo de sintomatología depresiva en adolescentes de sexo femenino. Terapia Psicológica, 27(2), 155-160. doi: 10.4067/S0718-48082009000200001

Cova, F., Rincón, P., Inostroza, C., \& Melipillán, R. (2012). Factores asociados prospectivamente a sintomatología depresiva en adolescentes tempranos de sexo femenino. Universitas Psychologica, 11(3), 853-862.

Del Pino, P. A., Ibáñez, F. I., Bosa, O. F., Dorta, G. R., \& Gaos, M. M. (2012). Modelos factoriales del Inventario de Depresión de Beck-II. Validación con pacientes coronarios y una crítica al modelo de Ward. Psicothema, 24(1), 127-132.

Dozois, D. J. A., Dobson, K. S., \& Ahnberg, J. L. (1998). A psychometric evaluation of the Beck Depression Inventory-II. Psychological Assessment, 10(2), 83-89.

Estrada, B. (2008). Salud mental: depresión y sordera. Monterrey, MX: Universidad Autónoma de Nuevo León.

Estrada, B, Delgado, C., \& Beyebach, M. (2010). Beck Depression Inventory-II in Spanish sign language. International Journal of Hispanic Psychology, 3(1), 25-46.

Estrada, B., \& Ruíz, I. R. (2012). Beck Depression Inventory-II in Mexican sign language. Reliability and factorial data. International Journal of Mental Health and Deafness, 1(2), 4-12.

Gallardo, I., \& Moyano-Díaz, E. (2012). Análisis psicométrico de las escala Ryff (versión española) a una muestra de adolescentes chilenos. Universitas Psychologica, 11(3), 931-939.

Hair, J. F., Anderson, R. E., Tatham, R. L., \& Black, W. C. (2001). Análisis Multiariante (5.a ed.). Madrid: Prentice-Hall.

Jakšić, N., Ivezić, E., Jokić-Begić, N., Surányi, Z., \& Stojanović-Špehar, S. (2013). Factorial and diagnostic validity of the Beck Depression Inventory-II (BDI-II) in Croatian primary health care. Journal 
of Clinical Psychology in Medical Settings, 20, 311322. doi: 10.1007/s10880-013-9363-2

Jöreskog, K. G., \& Sörbom, D. (1993). Structural equation modeling with the SIMPLIS command language. Chicago: Scientific Software International. Jurado, S., Villegas, M. E., Méndez, L., Rodríguez, F., Loperena, V., \& Varela, R. (1998). La estandarización del Inventario de Depresión de Beck para los residentes de la Ciudad de México. Salud Mental, 21(3), 26-31.

Kojima, M., Furukawa, T. A., Takahashi, H., Kawai, M., Nagaya, T., \& Tokudome, S. (2002). Cross-cultural validation of the Beck Depression Inventory-II in Japan. Psychiatry Research, 110(3), 291-299.

McDonald, R. P., \& Marsh, H. W. (1990). Choosing a multivariate model: Noncentrality and goodness of fit. Psychological Bulletin, 107(2), 247-255.

Montorio, I. (1990). Evaluación psicológica en la vejez: instrumentación desde un enfoque multidimensional (Tesis Doctoral no publicada). Madrid, Universidad Autónoma de Madrid.

Mulaik, S. A., James, L. R., Van Alstine, J., Bennett, N., Lind, S., \& Stilwell, C. D. (1989). Evaluation of goodness-of-fit indices for structural equation models. Psychological Bulletin, 105, 430-445. http:// dx.doi.org/10.1037//0033-2909.105.3.430

Muñiz, J., \& Fernández-Hermida, J. R. (2010). La opinión de los psicólogos españoles sobre el uso de los tests. Papeles del Psicólogo, 31(1), 108-121.

Novy, D. M., Stanley, M. A., Averill, P., \& Daza, P. (2001). Psychometric comparability of English- and Spanish-language measures of anxiety and related affective symptoms. Psychological Assessment, 13(3), 347-355. http://d.doi.org/10.1037/1040. 3590.13.3.347

Piotrowski, C., \& Keller, J. W. (1992). Psychological testing in applied settings: A literatura review from 1982-1992. Journal of Training and Practice in Professional Psychology, 6(2), 74-82.

Penley, J. A., Wiebe, J. S., \& Nwosu, A. (2003). Psychometric properties of the Spanish Beck Depression Inventory-II in a medical sample. Psychological Assessment, 15(4), 569-577. http://dx.doi. org/10.1037/1040-3590.15.4.569

Piotrowski, C., Sherry, D., \& Keller, J. W. (1985). Psychodiagnostic test usage: A survey of the Society for
Personality Assessment. Journal of Personality Assessment, 49(2), 115-119.

Raykov, T., \& Marcoulides, G. A. (2011). Introduction to psychometric theory. New York: Routledge.

Sanz, J. (2011). La adaptación española del Inventario de Depresión de Beck-II (BDI-II): Consejos para su utilización en investigación y en la práctica clínica. $\mathrm{V}$ Congreso Internacional y X Nacional de Psicología Clínica, Universidad Complutense, Madrid. Disponible en http://www.pearsonpsychcorp.es/ archivos/ 13373230291.pdf

Sanz, J., \& García-Vera, M. P. (2009). The Beck Depression Inventory (BDI-II) (2nd. ed.). School and Health, 21, 331-342.

Sanz, J., García-Vera, M., Espinosa, R., Fortín, M., \& Vázquez, C. (2005). Adaptación española del Inventario para la Depresión de Beck-II (BDI-II): 3. Propiedades psicométricas en pacientes con trastornos psicológicos. Clínica y Salud, 16(2), 121-142.

Sanz, J., Navarro, M., \& Vázquez, C. (2003). Adaptación española del Inventario para la depresión de Beck-II (BDI-II): 1. Propiedades psicométricas en estudiantes universitarios. Análisis y Modificación de Conducta, 29(124), 239-288.

Sanz, J., Perdigón, A., \& Vázquez, C. (2003). Adaptación española del Inventario para la Depresión de Beck-II (BDI-II): 2. Propiedades psicométricas en población general. Clínica y Salud, 14(3), 249-280.

Sanz, J., \& Vázquez, C. (1998). Fiabilidad, validez y datos normativos del Inventario para la Depresión de Beck. Psicothema, 10(2), 303-318.

Sierra, H. C. (2004). A study of the Spanish version of the Beck Depression Inventory-II. (Tesis inédita de Maestría). University of British Columbia. Vancouver, Canadá.

Song, Y. M., Lee, H. K., Kim, J. W., \& Lee, K. (2012). Reliability and validity of the Korean Version of Beck Depression Inventory-II via the internet: Results from a university student sample. Journal of Korean Neuropsychiatry Association, 51(6), 402-408.

Steer, R. A., \& Clark, D. A. (1997). Psychometric characteristics of the Beck Depression Inventory-II with college students. Measurement and Evaluation in Counseling and Development, 30(3), 128-136. 


\section{Benito Daniel Estrada Aranda, Carmen Delgado Álvarez, \\ René landero Hernández, Mónica Teresa González Ramírez}

Steer, R., Ball, R., Ranieri, W. F., \& Beck, A. T. (1999). Dimensions of the Beck Depression Inventory-II in clincally depresed outpatients. Journal of Clinical Psychology, 55(11), 117-128.

Suárez-Mendoza, A. A., Cardiel, M. H., Caballero-Uribe, C. V., Ortega-Soto, H. A., \& Marquez-Marin, M. (1997). Measurement of depression in Mexican patients with rheumatoid arthritis: Validity of the Beck Depression Inventory. Arthrithis Care and Research, 10(3), 194-199.

Torres-Castillo, M., Hernández-Malpica, E., \& OrtegaSoto, H. A. (1991). Validez y reproducibilidad del Inventario para la Depresión de Beck en un hospital de cardiología. Salud Mental, 14(2), 1-6.

Vázquez, C., \& Sanz, J. (septiembre, 1991). Fiabilidad y validez factorial de la versión española del Inventario de Depresión de Beck. Comunicación presentada en III Congreso de Evaluación Psicológica, Barcelona. Vázquez, C., \& Sanz, J. (1997). Fiabilidad y valores normativos de la versión española del Inventario para la Depresión de Beck de 1978. Clínica y Salud, 8(3), 403-422.
Vázquez, C., \& Sanz, J. (1999). Fiabilidad y validez de la versión española del Inventario para la Depresión de Beck de 1978 en pacientes con trastornos psicológicos. Clínica y Salud, 10(1), 59-81.

Whisman, M. A., Perez, J. E., \& Ramel, W. (2000). Factor structure of the Beck Depression Inventory-Second Edition (BDI-II) in a student sample. Journal of Clinical Psychology, 56(4), 545-551. http://dx.doi.org/10.1002/(SICI)10974679(200004)56:4<545::AID-JCLP7>3.0.CO;2-U

Wilson, R. C., \& Blumentritt, L. T. (2007). Psychometric properties of the Beck Depression Inventory-II in a clinically-identified sample of Mexican American adolescents. Journal of Child and Family Studies, 16, 789-798. http://dx.doi.org/10.1007\%2Fs10826006-9125-y

Wiebe, J. S. \& Penley, J. A. (2005). A Psychometric Comparison of the Beck Depression Inventory-II in English and Spanish. Psychological Assessment, $17,481-485$ 